\title{
MUSICAL INSTRUMENT RECOGNITION TECHNIQUES
}

\author{
A. S. Patankar \\ International institute of information technology,Hinjewadi \\ patankar.kargi@gmail.com
}

\section{Abstract}

Musical instrument recognition has become very popular and interesting field in recent years. There are many types of features used for musical instrument recognition e.g. temporal features, energy features, spectral features. We can represent music signal in time, frequency as well as log frequency domain. Wavelet transform is recently used feature extraction technique for musical instrument recognition. This paper reviews different techniques used in musical instruments identification. A comprehensive analysis is given along with classification detail to summarize the techniques.

Keywords: Musical instruments recognition, Feature extraction, Monophonic music.

\section{Introduction}

Automatic sound source recognition plays an important role in developing automatic indexing and database retrieval applications. These applications have potential in saving the humans from time taking searches through huge amounts of digital audio material available today. For instance, it would be most useful if we could find sound samples that sound similar as a given sound example. Music content analysis in general has many practical applications, including e.g. structured coding, automatic musical signal annotation, and musicians tools. Automatic musical instrument recognition is a crucial subtask in solving these difficult problems, and may also provide useful information in other sound source recognition areas, such as speaker recognition. However, musical signal analysis has not been able to attain as much commercial interest as, for instance, speaker and speech recognition. This is because the topics around speech processing are more readily commercially applicable, although both areas are considered as being highly complicated. Through constructing computer systems that listen, we may also gain some new insights into human perception. Recently, music data analysis and retrieval has become an emerging research field in signal processing. Due to tremendous applications of musical instruments, musical instrument recognition(MIR) has attracted the attention of various researchers.

In addition to this, the advancement in digital signal processing and data mining techniques has led to intensive study on music signal analysis like, content-based music. retrieval, music genre classification, duet analysis, Musical transcription , Musical Information retrieval and musical instrument detection and classification. Musical Instrument detection techniques have many potential applications such as detecting and analyzing solo passages, audio and video retrieval, music transcription, playlist generation, acoustic environment classification, video scene analysis and annotation etc.

\section{Literature Survey:}

Authors Athanasia Zlatintsi ,Petros Maragos, et al. had used Multi scale fractal dimension (MFD) outline as a short-time descriptor.This is useful to quantify the multi scale complication and fragmentation of the various states of the music waveform. In this work, method and features proposed are promising for music signal analysis. These methods and features are capable for multi scale analysis of the musical signals. This is applicable for musical recognition system. The results of this method are interesting and are applicable for classification of large scale music. Authors investigate the feature set which measures structures of music signals for multiple time scales[1].

Eronen presented a musical instrument recognition system using 32 spectral and temporal features. 1498 samples are taken to classify 30 orchestral instruments. Samples are taken from Mc Gill University Master Samples CD's. Gaussian and k-NN classifiers are used for classification. Accuracy of system was 95\% for group of instruments and $81 \%$ for individual instrument. $20 \mathrm{~ms}$ frames are used to calculate spectral feature[2].

Arie A. livshin et al. proposed a method in which a number of solo recordings are fed as training set Different features say, temporal, energy, spectral, harmonic and perceptual are extracted using Gradual descriptor Elimination (GDE) feature selection algorithm. The reduction of feature is done by Linear Discriminant Analysis (LDA). This is a real-time solo-recognition technique used $\mathrm{k}-\mathrm{NN}$ with LDA as classifier. The recognition score is $84.6 \%$. The problem arises in this technique is the classification of only 
single sound at a time. There is a need to recognize multi instrumental music[3].

An experimental study on feature analysis for recognition of musical instrument with the help of k-NN, Artificial Neural Network , and Support Vector Machine(SVM)classifier have been performed by J. D. Deng, C.Simmermacher. They used perception based features, MPEG-7 timbral feature and MFCC features[4].

Brown distinguished between oboe and saxophone by calculating cepstral coefficients and applying a k-means algorithm to form clusters[5].

Tao Li and Qi Li proposed features based on wavelet coefficients at various frequency sub bands of daubechies wavelet for music genre classification and emotional content of the music. The author used features like timbral texture,rhythmic content, pitch content, spectral centroid, and roll off,zero crossing, LPC, MFCC, spectral flux. The author claimed the result up to 80 percent[6].

G. Tzanetaki proposed a new technique in music genre classification. Three feature sets for representing timbre, texture, rhythmic and pitch content are proposed by this author. Using the proposed feature sets, musical genre classification of 61 percent was achieved for ten instruments. The success of proposed features for musical genre classification testi_es to their potential as the basis for other types of automatic techniques for music signals such as similarity retrieval and segmentation which are based on extracting features to describe musical content[7].

I. Kaminskyj and C. Pruysers described the technique to improve the Musical Instrument Recognition by adding Wavelet packet based features. The author claimed that the recognition accuracy is improved by _ve percentages by adding Morlet and Daubechies features. Using the proposed feature sets classification accuracy of 87.6 percent was claimed for 19 musical instruments. Specified work is focused on measuring the generalization performance of the system of monophonic instrument[8].

Eronen and Klapuri examined a wide range of temporal and spectral features from a large variety of orchestral instruments[9].

Popescu, Gavat and Mithai Datcu suggested a technique of Wavelet analysis for audio signals with music classification application. They used multi-resolution (wavelet) analysis and spectral

analysis based features. The Proposed approach uses a no. of features like Mel Frequency Cepstral Coefficient (MFCC), Zero Crossing rate and FFT Coefficients combined with wavelet based features. Using the proposed features the accuracy of 75 percent was claimed[10].

Cyril Joder and Slim Essid described temporal integration for audio classification with application to musical classification. Author compared the accuracy using different classifiers like HMM, GMM and DTW. Using the proposed methods the accuracy was claimed to 75 percent. In The paper suggested that the further work can be extended in designing automatic indexing tools which provide meaningful and efficient means to describe the musical audio content and also can be used in music information retrieval systems[11].

T. Kithara et al. proposed a pitch dependent musical instrument identification method. They used an FO dependent multivariate normal distribution. 129 features are extracted and then PCA and LDA technique are used for dimension reduction. Different parameters i.e. FO dependent multivariate normal distribution, FO dependent mean function and FO normalized covariance are calculated. Further, Bayes decision rule is used for

classification. The recognition rate for individual instrument is 75.73 to $79.93 \%$ and for category level is 88.20 to $90.65 \%[12]$.

Training Phase

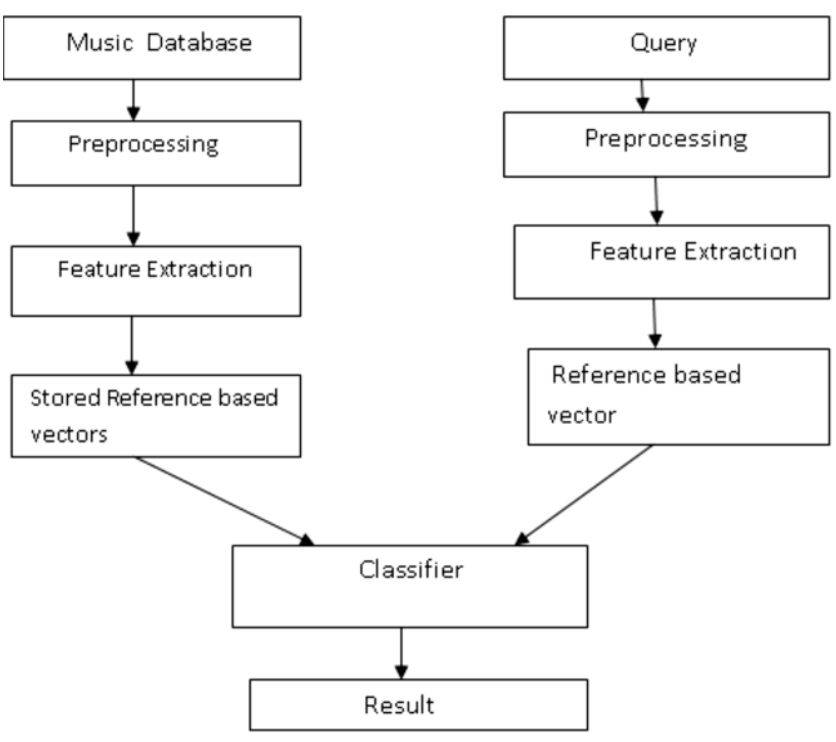

Figure: General block diagram of Musical Instrument Recognition 
Table 1. Comprehensive Analysis

\begin{tabular}{|c|c|c|c|}
\hline Authors & Features & $\begin{array}{l}\text { Feature } \\
\text { extraction } \\
\text { Techniques }\end{array}$ & $\begin{array}{l}\text { Classification } \\
\text { Techniques }\end{array}$ \\
\hline Eronen[2] & Temporal and Spectral feature & - & Gaussian, k-NN \\
\hline $\begin{array}{l}\text { Arie A. livshin et } \\
\text { al.[3] }\end{array}$ & Temporal, Energy, Spectral, Harmonic, Perceptual & LDA & $\mathrm{k}-\mathrm{NN}$ \\
\hline $\begin{array}{l}\text { J. D. Deng et } \\
\text { al.[4] }\end{array}$ & $\begin{array}{l}\text { Zero Crossing, Root Mean Square,SpectralCentroid, } \\
\text { Bandwidth,Flux, Harmonic Centroid, Harmonic } \\
\text { Deviation, Harmonic Spread, } \\
\text { Harmonic Variation, Spectral Centroid, Log Attack } \\
\text { time, Temporal } \\
\text { Centroid, MFCC }\end{array}$ & PCA, Isomap & $\mathrm{k}-\mathrm{NN}, \mathrm{RBF} \& \mathrm{SVM}$ \\
\hline Brown[5] & Cepstral & $\begin{array}{l}\mathrm{K} \text { means } \\
\text { algorithm }\end{array}$ & \\
\hline $\begin{array}{l}\text { Tao Li and Qi Li } \\
\text { [6] }\end{array}$ & $\begin{array}{l}\text { timbral texture, rhythmic content, } \\
\text { pitch content, spectral centroid and roll off, zero } \\
\text { crossing, LPC, MFCC, spectral }\end{array}$ & $\begin{array}{l}\text { Wavelet } \\
\text { transform } \\
\text { daubechies } \\
\text { wavelet }\end{array}$ & - \\
\hline Cyril Joder[11] & Temporal & - & $\begin{array}{l}\text { HMM, GMM and } \\
\text { DTW }\end{array}$ \\
\hline $\begin{array}{l}\text { Popescu,et } \\
\text { al.[10] }\end{array}$ & MFCC,Zero crossing rate & $\begin{array}{l}\text { Wavelet } \\
\text { analysis, } \\
\text { Spectral } \\
\text { analysis }\end{array}$ & - \\
\hline $\begin{array}{l}\text { I. Kaminskyj et } \\
\text { al.[8] }\end{array}$ & Wavelet based features & - & - \\
\hline $\begin{array}{l}\text { T.Kitahara et } \\
\text { al.[12] }\end{array}$ & Peak kurtosis, Modulation features,Temporal feature & PCA,LDA & $\begin{array}{l}\text { Bayes decision } \\
\text { rule }\end{array}$ \\
\hline
\end{tabular}

\section{Conclusion}

This paper concludes that the different features like temporal, spectral, and perceptual and energy extraction techniques are having major role in the concerned field. Extracting relevant information requires different kind of specialized method that is discussed in this paper. This paper presents different types of classification schemes used to identify musical instruments. It is clear that these techniques are not verge of the story of musical instrument recognition.

\section{References}

[1] Athanasia Zlatintsi, Student Member, IEEE, and Petros Maragos, Fellow, IEEE- Multiscale Fractal Analysis of Musical Instrument Signals With Application to Recognition.

[2] A. Eronen, A. Klapuri, Musical instrument recognition using Cepstral Coefficient and Temporal Feature, IEEE proceedings on International Conference on Acoustic, Speech and Signal Processing vol. 2, pp. 753-756, 2000.

[3] A. A. Livshin, and X. Rodet, Musical instrument identification in continuous recordings, in Proc. Int. Conf. Digital Audio Effects, , Italy, 2004.

[4] J. D. Deng, C. Simmermacher, and S. Crane_eld, A study on feature analysis for musical instrument classification, IEEE Trans. On System, Man, and Cybernetics- part B: cybernetics, Vol. 38, no. 2,April 2008.

[5] Brown, J.: Computer Identification of Musical Instruments Using Pattern Recognition with Cepstral Coefficients as Features. J. Acoust. Soc. Am. 105, 1933- 1941(1998).
[6] T. Li, Q. Li, and M. Ogihara, Music feature extraction using wavelet coefficient histograms, US Patent 7,091,409 B2, 2006.

[7] G. Tzanetakis, S. Member, and P. Cook, Musical Genre Classification of Audio Signals, IEEE Transactions on Audio, Speech and Language Processing, vol. 10, no. 5, pp. 293302, 2002.

[8] C. Pruisers,I. Kaminskyj and Schnapp, Wavelet analysis in musical instrument sound classification, in international symposium on signal processing and its applications, pp. 14, 2005.

[9] Eronen, A., Klapuri, A.: Musical Instrument Recognition Using Cepstral Coefficients and Temporal Features. In:IEEE International Conference on Acoustics, Speech and Signal Processing, pp. 753-756 (2000).

[10] M. Popescu, A.; Gavat, I.; Datcu, Wavelet analysis for audio signals with music classification applications, in proc. of speech technology and human computer dialogue, pp. 16.,2009.

[11] C. Joder, S. Essid, G. Richard, and S. Member, Temporal Integration for Audio Classification With Application to Musical Instrument Classification, IEEE Transactions on Speech and Audio Processing, vol. 17, no. 1, pp. 174186, 2009.

[12] T. Kithara, M. Goto, H. G. Okuno, "Pitch dependent identification of musical instrument sounds," Applied Intelligence, vol. 23, pp. 267275, 2005.

[13] "Speech and Audio Processing" by Dr. Shaila D.Apte. 